\title{
On the Performance of Counter-Based Broadcast Scheme for Mobile Ad Hoc Networks
}

\author{
${ }^{1}$ A. Mohammed and ${ }^{2 *}$ M.L. Jabaka \\ ${ }^{1}$ Department of Computing Science, University of Glasgow, Glasgow, G12 8RZ, UK \\ ${ }^{2}$ Department of Computer Science, Faculty of Management Studies and Information Technology, \\ Jamia Hamdard, Hamdard Nagar, New Delhi - 110062 India \\ *Corresponding Author: muhammadLawali@yahoo.com
}

\begin{abstract}
Broadcasting is a commonly used operation in mobile ad hoc networks (MANETs), where a source node diffuses a message to all other nodes in the network. Flooding, a process in which each node retransmits every uniquely received packet exactly once is the simplest mechanism used for broadcasting in MANETs. Despite its simplicity, it can result in high redundant retransmission, contention and collision, a phenomenon collectively referred to as the broadcast storm problem. Counter-based broadcast scheme has been proposed to mitigate this inherent problem and it relies on counter threshold value for rebroadcast decision. Thus, the selection of an appropriate counter threshold-value is crucial to the performance of the scheme. However, previous studies have focused on determining an optimal counter threshold-value using a network setting with very low traffic load. In this paper, we investigate the effects of the counter threshold value on the performance of counterbased scheme in terms of number of retransmitting nodes, reachability and collision rate, using Ns-2 simulation under varying network density and traffic load. Simulation results have revealed that an optimal counter threshold for sparse networks is smaller than that of dense networks. Thus, while most previous studies have often used a single fixed value for the counter, this paper argues that the optimal counter threshold is network density dependent.
\end{abstract}

KEYWORDS: Broadcasting, Flooding, Broadcast Storm Problem, Counter-based, Collision rate, Reachability.

\section{INTRODUCTION}

Mobile ad hoc networks (MANETs) are special type of wireless networks that encompasses a collection of wireless mobile nodes forming a temporary network without the aid of any centralized administration or standard support services(Basagni et al., 2004). It enables wireless communications between participating mobile devices with the assistance of other mobile devices. In such a network, each mobile node operates not only as a host but also as a router so that it can send and receive messages as well as forward messages for others. Scenarios that might benefit from MANETs technology include rescue/emergency operations in natural or environmental disaster areas, special operations during law enforcement activities, tactical missions in hostile and/or unknown territories, and commercial/academic gatherings such as conferences, exhibitions and workshops(Basagni et al., 2004).

In MANETs, broadcasting plays a crucial role as a means of diffusing a message from a source node to all other nodes in the network. It is a fundamental operation which is extensively used in route discovery, address resolution and many other network services in a number of routing
protocols(Colagrosso, 2007). For example Dynamic Source Routing (DSR)(Johnson and Maltz, 1996), Ad hoc On Demand Distance Vector (AODV)(Perkins and Moyer, 1999), Zone Routing Protocol (ZRP)(Haas et al., 1999.) and Location Aided Routing (LAR)(Ko and Vaidya, 1998) use broadcasting or its derivative to establish routes. Other routing protocols, such as the Temporally Ordered Routing Algorithm (TORA)(Park and Corson, July 2001) use broadcasting to transmit an error packet for invalid routes. These protocols typically assume a simplistic form of broadcasting known as flooding, in which each mobile node retransmits every unique received packet exactly once. Although flooding achieves high success rate in reaching all nodes in the network, it produces redundant rebroadcast messages. In dense network, this redundant rebroadcast can often cause high contention and collision in the network, leading to loss of precious bandwidth and battery power, a phenomenon called broadcast storm problem(Wu and Lou, 2003).

To mitigate this problem, several broadcast schemes have been proposed( Stojmenovic and Zunic, 2002; Wu and Lou, 2003, Rogers and 
Abu-Ghazaleh, 2004, Keshavarz-Haddad et al., 2006,). These schemes are commonly divided into two categories: deterministic schemes $(\mathrm{Wu}$ and Lou, 2003, Rogers and Abu-Ghazaleh, 2004) and probabilistic schemes(Keshavarz-Haddad et al., 2006, Zhang and Agrawal, 2005). Deterministic schemes use network topological information to build a virtual backbone that covers all the nodes in the network(KeshavarzHaddad et al., 2006). To build a virtual backbone, nodes exchange information about their immediate or two hop neighbours. However, this often incurs large overhead in terms of time and message complexity for building and maintaining the backbone, especially in the presence of mobility. Probabilistic schemes, in disparity, rebuild a backbone from scratch during each broadcast(Zhang and Agrawal, 2005.). Nodes make instantaneous local decisions about whether to broadcast a message or not using information derived only from overheard broadcast messages. Consequently, these schemes incur a smaller overhead and demonstrate superior adaptability in changing environments when compared to deterministic schemes (Keshavarz-Haddad et al., 2006). However, these schemes have poor reachability as a trade-off against overhead.

An efficient broadcast should be able to minimize the number of retransmissions without sacrificing reachability or having any significant degradation. In this paper, we examined counterbased broadcast scheme as one of the proposed probabilistic schemes in literature that mitigate the broadcast storm problem inherent with flooding. Counter-based broadcast schemes for MANETs have been first proposed in ( $\mathrm{Ni}$ et al., 1999) and further investigated in (Tseng et al., 2002, Tseng et al., 2003, Zhang and Agrawal, 2005). In counter-based schemes, every mobile node relies on a predetermined counter thresholdvalue $(C)$, to decide whether or not to rebroadcast a packet. These schemes do not require global topological information of the network in order to make rebroadcast decision. Thus, these schemes are localized and can considerably reduce the number of retransmission predominant in flooding but on the other hand cannot guarantee full network coverage in sparse network. One major challenge in counter-based schemes is how to select an appropriate $C$ that can optimize the performance of counter-based scheme in terms of number of retransmitting nodes, reachability and end-to-end delay. Most counter-based schemes assume a counter threshold-value of 3 or 4 (Zhang and Agrawal, 2005., Keshavarz-Haddad et al., 2006). It has been shown that a thresholdvalue of 3 or 4 can save many rebroadcasts in a dense network while achieving a reachability ratio comparable to flooding (Ni et al., 1999). On the other hand, a larger threshold of $C>6$ will provide less saving of rebroadcasts in a sparse network but behave almost like flooding in terms of reachability. However, previous studies ( $\mathrm{Ni}$ et al., 1999, Tseng et al., 2003) had determined the threshold-value in the context of light traffic injection rate scenario.

Several previous studies (Williams and Camp, 2002; Tseng et al., 2003; Zhang and Agrawal, 2005) have used the threshold value suggested by (Ni et al., 1999). Nevertheless, these studies have assumed different traffic conditions (i.e. 10 packets per second and above) that have not been considered by (Ni et al., 1999). This paper investigated the effect of different counter threshold values on the performance of counterbased broadcast schemes in MANETs under a wide range of traffic conditions (i.e. low, moderate and high traffic loads) and network density. Moreover, we will show that when factors such as traffic and density are taken into account, the optimal counter threshold value can be different from that reported by Ni et al. (1999).

\section{Counter-Based Broadcast Scheme}

In this section, we discuss the counter-based broadcast scheme that aims to mitigate the broadcast storm problem associated with flooding. The use of the scheme for broadcasting in MANETs enables mobile nodes to make localized rebroadcast decisions.

Ni et al. (1999) have shown an inverse relationship between the number of times a packet is received at a particular node and the probability of that node being able to reach additional coverage area on a rebroadcast. This result is the foundation of their counter-based broadcast scheme. Specifically, a node upon reception of a previously unseen packet initiates a counter $c$ that will record the number of times a node receives the same packet. Such a counter is maintained by each node for each broadcast packet. After waiting for a random assessment delay (RAD, which is randomly chosen between 0 and $T_{\max }$ seconds), if $c$ reaches a predefined threshold $C$, the packet is rebroadcasted. Each node increments its $c$ by one each time it receives the same packet until the RAD expires. The node compares its $c$ with a predefined counter threshold $C$. If $c<C$, the node 
rebroadcasts the packet. Otherwise the packet is dropped. The scheme can be summarized in Fig. 1.

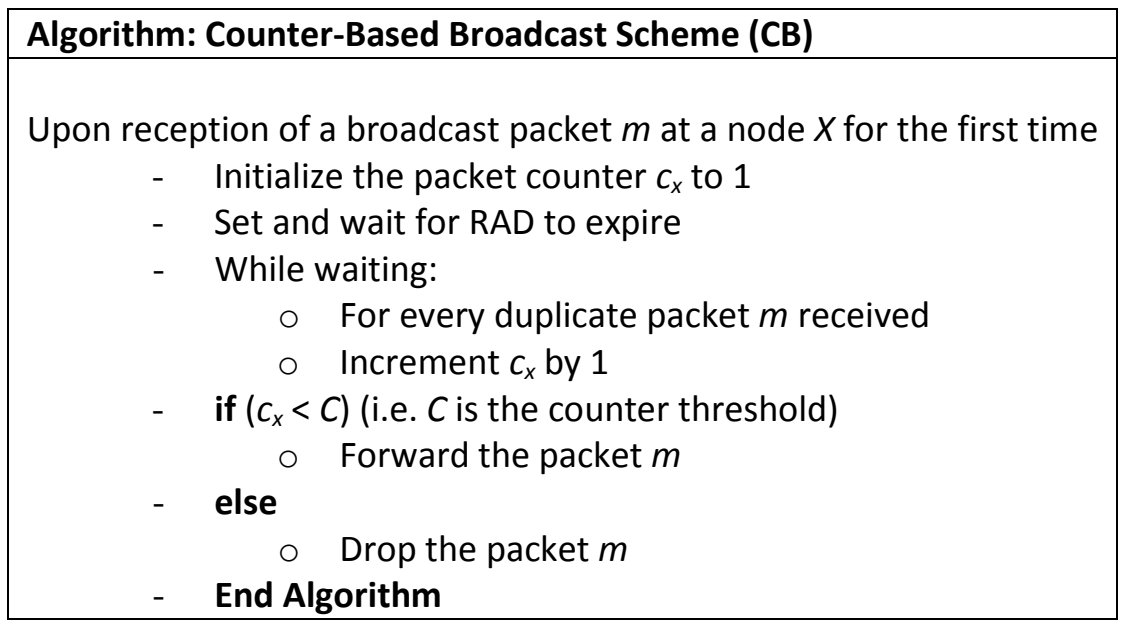

Figure 1: A description of the counter-based broadcast scheme

\section{Related Work}

Counter-based scheme was introduced after analysing the additional coverage of each rebroadcast when receiving $n$ copies of the same packet(Ni et al., 1999). The predefined threshold $\mathrm{C}$ is the key parameter in this approach. They showed that about $67 \%$ of the rebroadcasts could be saved when choosing a $\mathrm{C}$ value of 3 or 4 while the amount of saving decreases sharply if $\mathrm{C}>6$, especially in sparse network. In their follow-on work Tseng et al. (2003) proposed an adaptive counter-based scheme in which each node can dynamically adjust its threshold value $C$ based on its number of neighbours. Specifically, they extend the fixed threshold $C$ to a function $C(n)$, where $n$ is number of neighbours of the node. This approach requires a neighbour discovery mechanism to estimate the current value of $n$ which can be achieved through periodic exchange of 'Hello' packets among mobile nodes.

Zhang and Agrawal (2005) described a dynamic probabilistic broadcast scheme which is a combination of the probabilistic and counterbased approaches. The scheme is implemented for route discovery process using AODV as base routing protocol. The rebroadcast probability $P$ is dynamically adjusted according to the value of the local packet counter at each mobile node. Therefore, the value of $P$ changes when the node moves to a different neighbourhood. The packet counter is used as density estimates (i.e. a high value implies that the number of neighbours is high, and a low value corresponds to a small number of neighbours). This scheme uses the counter threshold value suggested by Ni et al. (1999), however, a different traffic rate of 10 packet per second was used for the evaluation of the scheme.

In Chen et al.( 2005), a distance-aware counterbased broadcast scheme called "DIS_RAD" has been suggested that introduced the concept of distance into counter-based broadcast scheme. The scheme gives nodes closer to the border of the transmission range a higher rebroadcast probability because they can have a high chance of reaching more nodes. A distance threshold is employed to distinguish between interior and border nodes using two distinct RAD values with the border nodes having shorter RADs than the interior nodes. This simple adaptation provides border nodes with higher rebroadcast probability and a lower rebroadcast probability for the interior nodes. Although the approach has superior performance over traditional counter-based scheme it suffers from the limitation of all distance-based schemes (i.e. determination of location information and optimal threshold value). Moreover, the same counter threshold was used as in the previous scheme which might not be optimal in the considered settings.

Previous work by Keshavarz-Haddad et al.(2006) proposed a colour-based broadcast scheme in which every broadcast message has a colour-field, with a rebroadcast condition to be satisfied after expiration of the timer similar to counter-based scheme. A node rebroadcasts a message with a new colour assigned to its colour-field if the number of colours of broadcast messages overheard is less than a colour threshold $\mu$. 
Recently, an enhanced counter-based scheme (ECS) (Mohammed et al., 2007), was proposed which combines the merits of probability-based and counter-based algorithms. The scheme enables mobile nodes to make localized rebroadcast decisions on whether or not to rebroadcast a message based on counter threshold and forwarding probability values of around 0.5 to yield a better performance in terms of saved-rebroadcast, end-to-end delay and reachability. However, its use of single fixed forwarding probability for all nodes in the network regardless of whether the node is in sparse or dense region of the network made it inflexible in a typical MANET scenario where regions of varying node density co-exist in the same network.

In their follow-on work a new adjusted counterbased broadcast scheme (ACBS) (Mohammed et al., 2008) is proposed, which uses two rebroadcast probabilities to differentiate between mobile nodes in sparse and dense area. However, most of these proposed counter-based broadcast schemes used the threshold value suggested in (Ni et al., 1999) for the evaluation of their scheme which might not be optimal in their network settings.

\section{Performance Analysis}

This section describes the details of our simulation environment, performance metrics used in our analysis and finally simulation results.

\section{Simulation environment and metrics}

The nature of MANETs makes simulation an invaluable tool for understanding the operation of these networks. As wireless channels experience high variability in quality due to multi-path, fading, atmospheric effects, or obstacles, while real world tests (i.e. test beds or real life implementations) and analytical models are crucial for understanding the performance of mobile network protocols, simulation was selected as a method of study because it provides an environment which can allow repeatable scenario evaluation, isolation of parameters and wide variety of scenarios and network configuration evaluation on a reasonable scale, time and budget. Likewise a detailed complete analytical model for multihop networks with reasonable assumptions is coarse in nature (Perrone et al., 2003). Therefore, the above characteristics are extremely difficult, if not impossible, with real world experiments. Due to these benefits, simulation has become a popular tool for the development and study of ad hoc networking protocols.

The study is conducted using Ns-2 simulator a discrete event simulator widely used in MANETs community (Broch et al., 1998, Kurkowski et al., 2005), to simulate a network of $1000 \times 1000 \mathrm{~m}^{2}$ area populated with 20,40 , $60, \ldots, 200$ mobile nodes. The simulation model consists of two set of scenario files: topology scenario and traffic generation files. The topology scenario files define the mobility model which governs the distribution of mobile nodes within the simulation area over the simulation period. On the other hand, the traffic pattern file contains information such as packet type, data packet size, broadcast packet origination rate and the number of traffic flows. In all scenarios, each node is assumed to be equipped with a wireless transceiver operating on IEEE $802.11 \mathrm{~b}$ wireless standard (1999). The physical radio characteristics of each node such as the transmitting power, signal to noise and interference ratio and antenna gain, are chosen to mimic the commercial Lucent's OriNOCO Wireless LAN PC Card (2000) with a nominal bit rate of $11 \mathrm{Mb} / \mathrm{s}$ and a transmission range of 100 meters with an Omni-directional antenna. To gain more realistic signal propagation than with the deterministic free space or two-ray ground reflection models (Sakar et al., 2003), the shadowing model is used as a radio propagation (Rappaport, 2001). The simulation is allowed to run for 900 seconds for each simulation scenario to avoid immature termination and to keep the simulation time manageable. The random trip mobility model (Boudec and Vojnovic, 2006) was used as the mobility model to generate 30 mobility topologies. Although, random way-point model (Boleng, 2001) is the most widely used mobility model but it suffers from speed decay problem, as such it takes more time to reach a stable distribution of mobile nodes. In random trip model, at a trip transition instant, a mobile node picks a trip destination uniformly at random within the area and samples numeric speed from a uniform distribution [minimum speed, maximum speed]. At the end of the trip, the mobile node picks another path according to the model's trip selection rule driven by a Markov chain. This cycle repeats until the end of the simulation time. Unlike other random mobility model, random trip node mobility 
distribution converges to a steady-state regime from origin of an arbitrary trip and there is no need to discard initial sets of simulation observations. Other simulation parameters that have been used in our experiment are shown in Table 1.

Table 1. Summary of Simulation Parameters

\begin{tabular}{|l|l|}
\hline Simulation Parameter & Value \\
\hline Simulator & $\mathrm{NS}-2(2.29 .3)$ \\
Transmission range & $100 \mathrm{~m}$ \\
Packet size & 512 bytes \\
Interface queue length & 50 \\
Topology size & $1000 \mathrm{~m} \times 1000 \mathrm{~m}$ \\
Number of nodes & $20,40, \ldots, 200$ \\
Simulation time & $900 \mathrm{~seconds}$ \\
Bandwidth & $11 \mathrm{Mbps}$ \\
Maximum speed & $1,5,10, \ldots .20 \mathrm{~m} / \mathrm{s}$ \\
Packet origination rate & $10,20, \ldots, 50$ packets $/ \mathrm{sec}$ \\
Number of trials & 30 \\
Confidence interval & $95 \%$ \\
MAC type & $802.11 \mathrm{~b}$ \\
Counter threshold & $2-6$ \\
Traffic type & Constant bit rate (CBR) \\
\hline
\end{tabular}

This paper focused on two major network operating conditions: Network density and traffic load, using two different cases by varying one condition while keeping the other constant in order to avoid the effect of one condition on the performance result of the varying condition. In both cases the mobility is assumed to be constant in order to avoid the effect of mobility in terms of frequent link breaks and also to focus on one category of node speed that can mimic a slow walking human (i.e. $2 \mathrm{~m} / \mathrm{s}$ ).

- Network Density: This refers to the total number of nodes in the network. It is used to study the effect of varying network density on the performance of the network. The simulation area is kept constant in all scenarios from sparse to dense network. Simulation has been performed by deploying $20,40,60, \ldots, 200$ nodes while fixing the maximum speed to $2 \mathrm{~m} / \mathrm{s}$ and the traffic load of 10 packet per second.

- Traffic Load: This is used to study the effect of varying the amount of traffic load on the performance of the network. Broadcast injection rate of 1 , $10,20,30, \ldots, 50$ packets per second were used while the network density is kept to 100 nodes to avoid sparse and dense scenarios with a maximum speed $2 \mathrm{~m} / \mathrm{s}$ to avoid the effect of mobility.
The performance of different threshold values is measured using the following performance metrics which have been widely used in the literature ( Broch et al., 1998; Ni et al., 1999, Tseng et al., 2002, Zhang and Agrawal, 2005; Bani-Yassein et al., 2006; Colagrosso, 2007,). Retransmitting nodes: The number of nodes in the network that receives a broadcast packet and rebroadcast it.

- Reachability $(R E)$ : The percentage of network mobile nodes that receive a given broadcast packet over the total number of nodes that is reachable, directly or indirectly.

- Collisions rate: The total number of control packets dropped by the MAC layer as a result of collisions per unit of the simulation time.

\section{SIMULATION RESULTS \& DISCUSSION}

This section presents the performance results of the two network operating conditions (i.e., density and load) on counter-based scheme over different threshold values. The simulation output is collected using replication mean method (Law 2008) where each data point represents an average of 30 different randomly generated mobility topologies using 95\% confidence intervals.

\section{Effect of Network Density}

\section{Performance Metrics}


The network density has been varied by deploying 20, 40, 60... 200 nodes over a network topology of $1000 \mathrm{~m} \times 1000 \mathrm{~m}$. Each node in the network moves according to random trip mobility model with minimum and maximum speeds of $1 \mathrm{~m} / \mathrm{s}$ and $2 \mathrm{~m} / \mathrm{s}$ respectively. In each simulation trial, a broadcast injection rate of 10 packets per second has been used with each new broadcast packet assigned a source node randomly chosen from the entire pool of network nodes in order to create a random traffic pattern.

\section{Number of Retransmitting Nodes}

Figure 2 shows the effects of density on the performance of different counter threshold values together with flooding in terms of number of retransmitting nodes. The figure reveals that the number of retransmitting nodes for a given threshold value increases with increasing network density. A low threshold value (i.e. $C=2$ ) requires least number of retransmissions while those utilising higher threshold values (i.e. $C=5,6$ ) require the largest number of rebroadcasts. In fact threshold values greater than 4 behave almost similar to flooding because most of the nodes retransmit the packets. For example in Figure 2, for a network of 100 nodes about $40 \%$ of the nodes retransmit for the threshold value 2 while around $98 \%$ of the nodes retransmit for threshold value 6 .

\section{Reachability}

Figure 3 depicts the reachability performance achieved by the different threshold values over a varying network density. The figure shows that reachability increases with increase in network density. For example, reachability achieved by threshold value 2 increases from $26 \%$ for 20 nodes to $98 \%$ for 100 nodes while that of threshold value 6 increases from $45 \%$ to 99.9\% for 20 and 100 nodes respectively. This is because as number of nodes increases there is more likelihood that nodes are located within the transmission range of each other and thus resulting in a better network connectivity. Similarly, the figure also reveals that low threshold value (i.e. $C=2$ ) achieves the least reachability in sparse to medium networks (20 to 80 nodes). But as the density increases reachability improves for all threshold values. As in Figure 2, for threshold values 4 and above, the counter-based scheme converges to flooding in terms of reachability performance. This is because the higher the threshold values, more nodes retransmit the broadcast packets. Therefore, to maintain a high reachability in sparse networks, a higher threshold value is required while to maintain reachability in dense networks, a low threshold value can be used. Thus, reachability improves with increased network density.

\section{Collision Rate}

Figure 4 shows that the collision rate for a given threshold value increases almost linearly as network density increases. This is due to the fact that increasing the network density increases the chances of two or more nodes within the same transmission range transmitting at the same time, leading to a possible increase in the number of collisions. The figure also reveals that for a given network size, the number of collision incurred by the different threshold values increases as the threshold value increases. As can be seen in Figure 4, for a network with 100 nodes, the collision rate for threshold value 3 increases by a factor of around 3 compared to threshold value 2 while the collision rate increases by a factor of 5 for threshold value 5 compared to threshold value 2. Similar to Figures 2 and 3, for threshold values of 4 and above, the counter-based scheme behave similar to flooding as most of the nodes are involved in packet retransmission. 


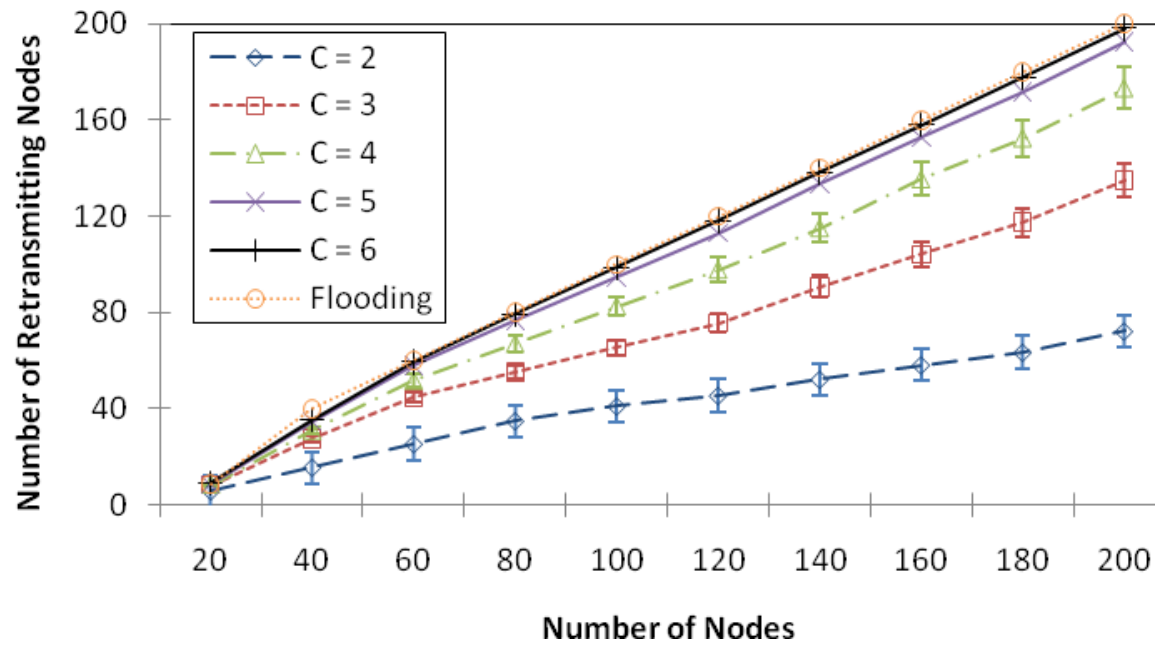

Figure 2: Number of retransmitting nodes vs. number of nodes placed over $1000 \mathrm{~m} \mathrm{x}$

$1000 \mathrm{~m}$ area when a broadcast rate of 10packets/sec is used for different threshold values.

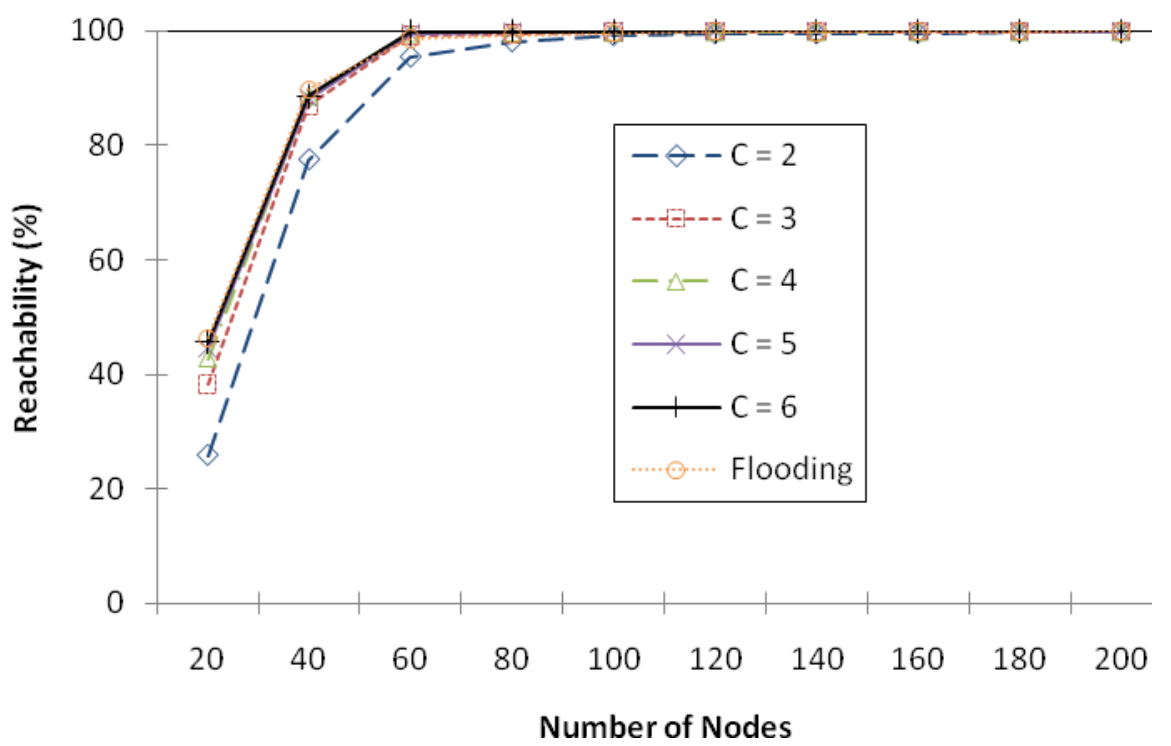

Figure 3: Reachability vs. number of nodes placed over $1000 \mathrm{mx} 1000 \mathrm{~m}$ area when a broadcast rate of 10packets/sec is used for different threshold values.

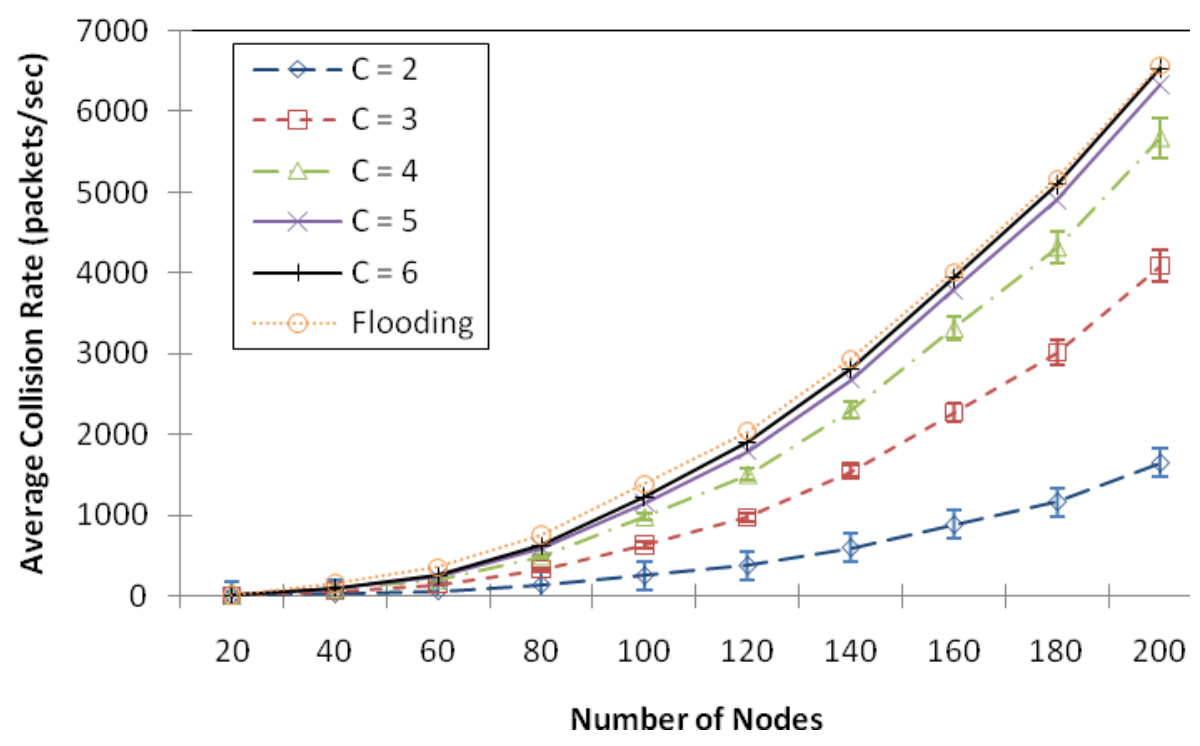


Figure 4: Average Collision rate vs. number of nodes placed over 1000mx1000m area when an injection rate of 10packets/sec is used for different threshold values.

\section{Effect of Traffic Load}

To investigate the impact of traffic load, the injection rates of $1,10,20,30,40$ and 50 packets per second have been used with each new broadcast packet assigned a source node randomly chosen from the entire pool of network nodes. 100 nodes are placed over a network topology of $1000 \mathrm{~m} \times 1000 \mathrm{~m}$ area and each node in the network moves according to random trip mobility model with minimum and maximum speeds of $1 \mathrm{~m} / \mathrm{s}$ and $2 \mathrm{~m} / \mathrm{s}$ respectively.

\section{Number of Retransmitting Nodes}

The results in Figure 5 show the effects of offered traffic load on the network performance for different threshold values in terms of number of retransmitting nodes. As expected, the number of retransmitting nodes for a given threshold value almost remain constant over different traffic loads. This is due to the use of fixed number of nodes (i.e. 100 nodes) in this simulation scenario. Nevertheless, a low threshold value (i.e. $C=2$ ) requires the least number of retransmissions while high threshold values (i.e. $C=5,6$ ) require the largest number of retransmissions. For example in the same figure, around $41 \%$ (41 nodes) of nodes retransmit when $C=2$ while about $65 \%$ of the nodes retransmit for $C=3$ and around $84 \%$, $94 \%$ and $98 \%$ of the nodes retransmit for $C=4$, 5 and 6 respectively. Therefore, the higher the threshold values the higher the number of retransmitting nodes.

\section{Reachability}

Figure 6 reveals that reachability decreases with increased broadcast injection rate, i.e. a heavier load will result in a lower reachability performance. This is true for all threshold values and flooding, because a high broadcast rate leads to more contention and collision among broadcast packets. For example, flooding is the most affected as reachability falls to around $85 \%$ at a broadcast rate of 50 packets/sec. Moreover, to maintain a better reachability a low threshold value is required especially in dense network. The figure also reveals that a low threshold value is advantageous when the injection rate is over 20 packets per second.

\section{Collision Rate}

The result in Figure 7 reveals that when the offered load increases, the average collision rate of all the threshold values and flooding also increases. This is because, when the injection rate is increased, the number of broadcast packet generated and transmitted also increases. Thus, the probability of two or more nodes transmitting at the same time within the same transmission range increases. This in turn leads to an increase in the collision rate. However, for a given injection rate, the average collision rate of the counter-based scheme with a threshold value 2 is much lower compared with the other threshold values and flooding. Similar to Figures 5 and 6, the figure also depicts that for threshold values greater that 4 the behaviour of the counter-based scheme converges to flooding. 


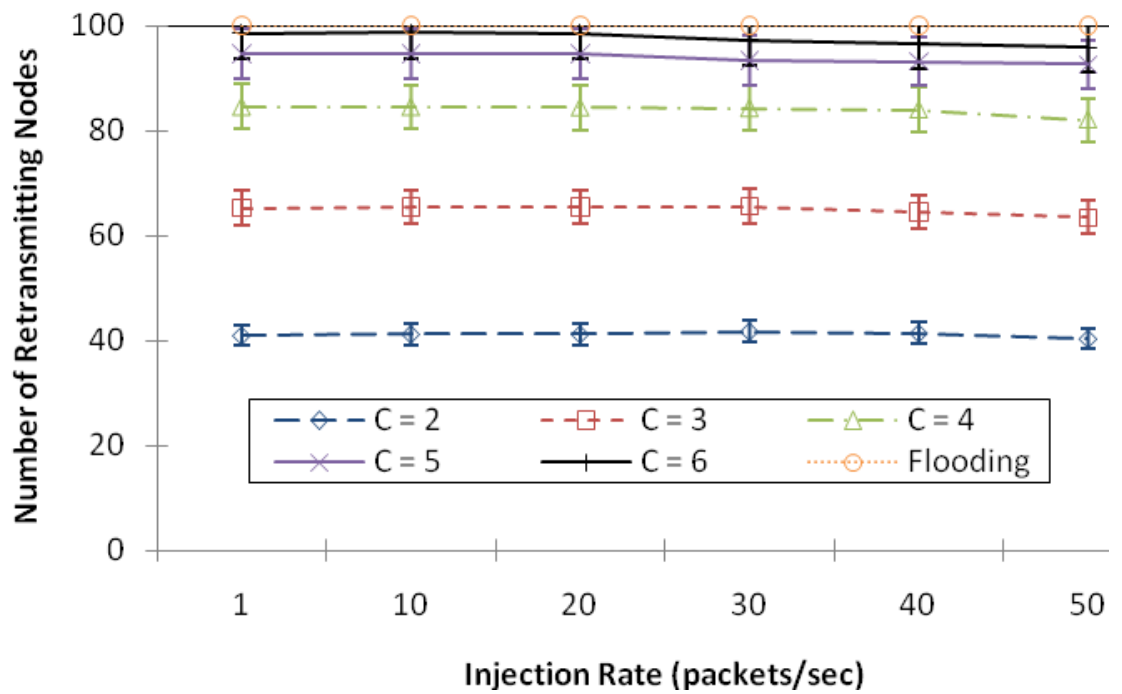

Figure 5: Number of retransmitting nodes vs. broadcast injection rates in a network of 100 nodes placed over $1000 \mathrm{mx} 1000 \mathrm{~m}$ area for different threshold values.

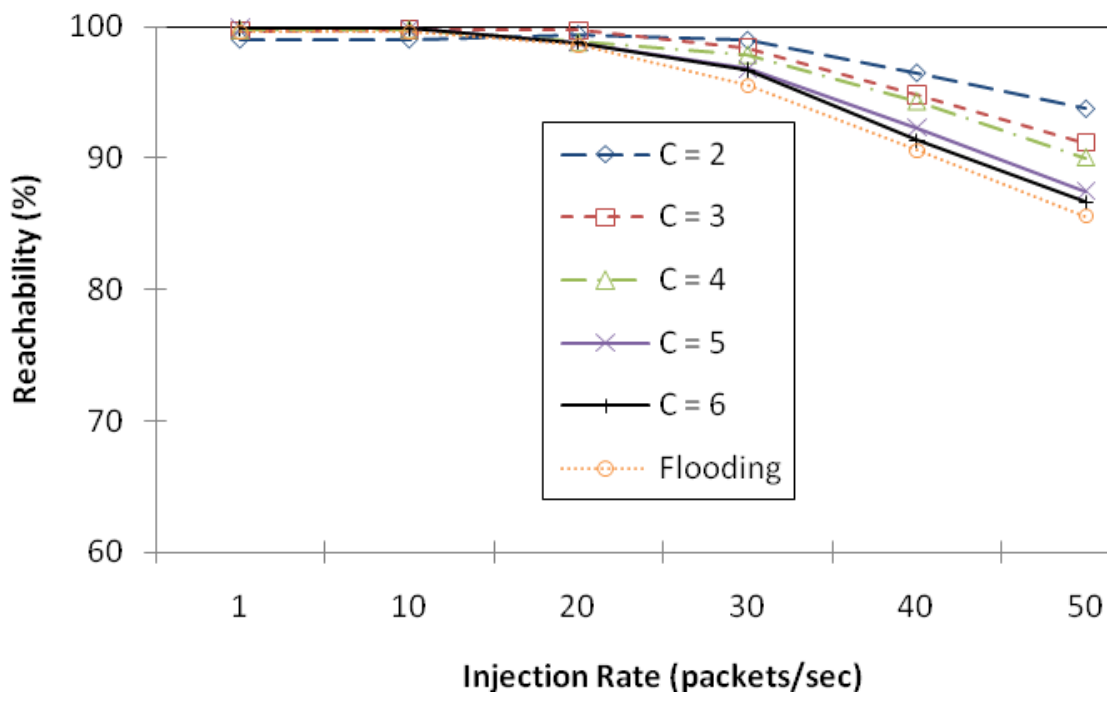

Figure 6: Reachability vs. broadcast injection rates in a network of 100 nodes placed over $1000 \mathrm{~m} \times 1000 \mathrm{~m}$ area for different threshold values.

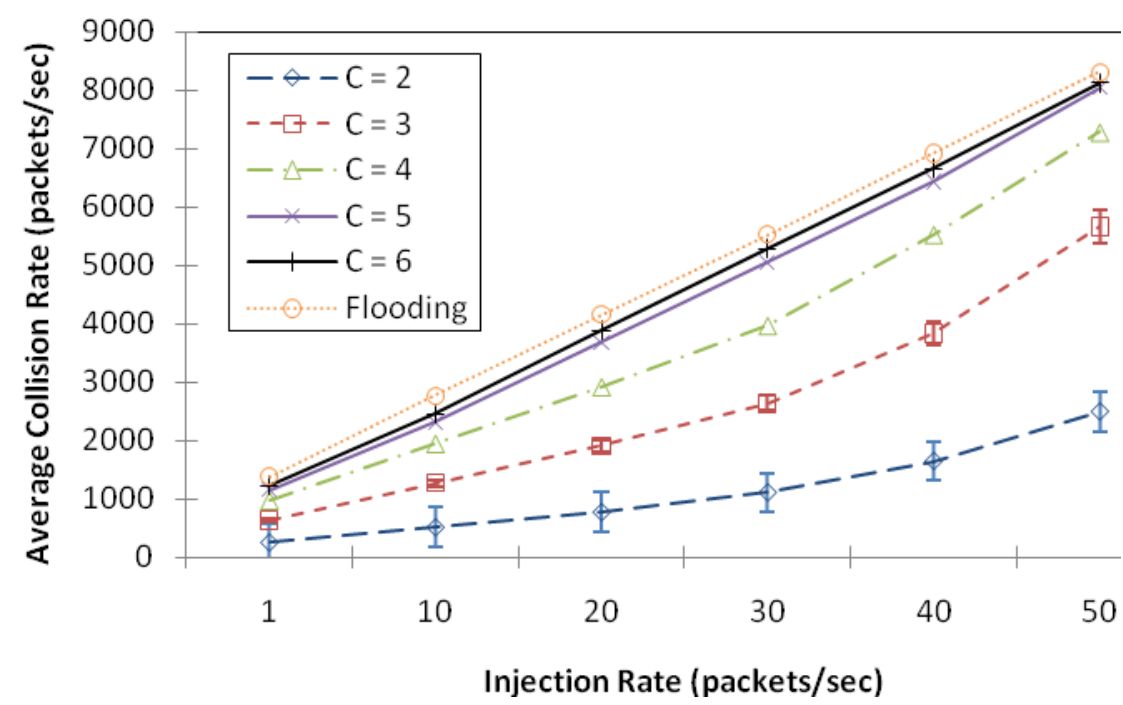

Figure 7: Average Collision rate vs. broadcast injection rates in a network of 100 nodes placed over $1000 \mathrm{~m} \times 1000 \mathrm{~m}$ area for different threshold values. 


\section{Conclusions}

Network density and traffic load over different counter threshold values. The results have revealed that network density and offered traffic load have significant impact on the performance of the scheme in terms of number of retransmitting nodes, reachability and collision rate. The selection of an appropriate threshold value dictates the achieved performance output of counter-based scheme. The performance of counter-based scheme can be enhanced by adapting the counter threshold values to local density. So that sparse and dense regions of the network can use different threshold values to improve performance. However, this adaptation requires the exchange of neighbourhood information between nodes using periodic exchange of "hello" packets. Similarly, the reachability performance of counter-based scheme suffers in congestive network irrespective of which threshold value is used. This is particularly due to the scheme's random assessment delay (RAD) mechanism. However, a possible solution to this problem is to devise a mechanism which can adapt node's RAD to its local congestion level.

\section{References}

Bani-Yassein, M., Ould-Khaoua, M., Mackenzie, L. M. and Papanastasiou, S. (2006) Performance Analysis Of Adjusted Probabilistic Broadcasting In Mobile Ad Hoc Networks. International Journal of Wireless Information Networks, 1-14.

Basagni, S., Conti, M., Giordano, S. and Stojmenovic, I. (2004) Mobile Ad Hoc Networking, Ieee Press.

Boleng, J. (2001) Normalizing Mobility Characteristics And Enabling Adaptive Protocol For Ad Hoc Networks. Proceedings Of The Ieee Local And Metropolitan Area Networks Workshop (Lanman).

Boudec, J.-Y. L. and Vojnovic, M. (2006) The Random Trip Model: Stability, Stationary Regime, And Perfect Simulation. Ieee/Acm Transactions On Networking, 14, 1153-1166.

Broch, J., Maltz, D. A., Johnson, D. B., Hu, Y.C. and Jetcheva, J. (1998) A Performance Comparison Of MultiHop Wireless Ad Hoc Network Routing Protocols. Proceedings of Acm/Ieee International Conference On
Mobile Computing And Networking (Mobicom'98), 85 - 97.

Chen, C., Hsu, C.-K. and Wang, H.-K. (2005) A Distance-Aware Counter-Based Broadcast Scheme For Wireless Ad Hoc Networks. Military Communications Conference (Milcom 2005) Ieee.

Colagrosso, M. D. (2007) Intelligent Broadcasting In Mobile Ad Hoc Networks: Three Classes Of Adaptive Protocols. Eurasip Journal On Wireless Communication And Networking, 2007, $25-40$.

Haas, Z. J., Pearlman, M. R. and Samar, P. (1999.) Determining Optimal Configuration For Zone Routing Protocol. Ieee Journal On Selected Areas In Communications, 17, 1395 1414.

Ieee (1999) Std 802.11b, Wireless Lan Medium Access Control (Mac) And Physical Layer (Phy) Specifications. HigherSpeed Physical Layer Extension In 2.4 Ghz Band. Supplement To Ieee 802.11 Standard.

Johnson, D.B. and Maltz, D. A. (1996) Dynamic Source Routing In Ad Hoc Wireless Networks. In Imelinsky, T. and Korth, H. (Eds.) Mobile Computing. Kluwer Academic Publishers.

Keshavarz-Haddad, A., Ribeiro, V. and Riedi, R. (2006) Color-Based Broadcasting For Ad Hoc Networks. Proceeding Of The 4th International Symposium On Modeling And Optimization In Mobile, Ad Hoc, And Wireless Network (Wiopt' 06). Boston, Ma.

Ko, Y.-B. and Vaidya, N. H. (1998) Location Aided Routing (Lar) In Mobile Ad Hoc Networks. Proceedings of The 4th Acm/Ieee International Conference On Mobile Computing And Networking (Mobicom). Dallas, Texas.

Kurkowski, S., Camp, T. and Colagrosso, M. (2005) Manet Simulation Studies: The Incredibles. Acm Sigmobile Mobile Computing And Communications Review, 9, 50 - 61.

Law, A. M (2008) Simulation Modeling And Analysis,New Delhi, Tata Mcgraw-Hill Publishing Company Limited.

Lucent Technology-Bell Labs Innovation, Orinoco Pc Card(2000) User's Guide. 
[Online] Available From:

Ftp://Ftp.Orinacowireless.Com/Pub/D

ocs/Orinoco/Manuals/Ug_Pc.Pdf.

Mohammed, A., Ould-Khaoua, M., Mackenzie,

L. M. and Abdulai, J. (2008) An

Adjusted Counter-Based Broadcast

Scheme For Mobile Ad Hoc Networks.

Proceedings Of The Tenth International

Conference On Computer Modeling

And Simulation(Eurosim/Uksim 2008).

Mohammed, A., Ould-Khaoua, M., Mackenzie,

L. M andAbdulai, J. (2007) Improving

The Performance Of Counter-Based Broadcast Scheme For Mobile Ad Hoc Networks. Proceedings of 2007 Ieee International Conference On Signal Processing And Communications (Icspc 2007).

Ni, S., Tseng, Y., Chen, Y. and Sheu, J. (1999), The Broadcast Storm Problem In A Mobile Ad Hoc Networks. Proceeding Of The Acm/Ieee International Conference On Mobile Computing And Networking (Mobicom).

Park, V. and Corson, S. (July 2001) Temporally-Ordered Routing Algorithm (Tora) Version 1. Iets Internet Darft (Draft-Ietfmanet-ToraSpec-04.Txt).

Perkins, C. E. and Moyer, E. M. (1999) Ad-Hoc On-Demand Distance Vector Routing. Proceedings Of 2nd Ieee Workshop On Mobile Computing Systems And Applications. New Orleans, L.A.

Perrone, L. F., Yuan, Y. and Nicol, D. M. (2003) Modeling And Simulation Best Practices For Wireless Ad Hoc Networks. Proceeding Of The 2003 Winter Simulation Conference. New Orleans, Louisiana, Ieee Press.

Rappaport, T. S. (2001) Wireless Communications: Principles And Practice.New Jersey, Prentice Hall Ptr Upper Saddle River.
Rogers, P. and Abu-Ghazaleh, N. (2004) Towards Reliable Network Wide Broadcast In Mobile Ad Hoc Networks.[Online],Available From: Http://Arxiv.Org/Abs/Cs/0412020.

Sakar, T. K., Ji, Z., Kim, K., Medourand, A. and Salazar-Palma, M. (2003) A Survey Of Various Propagation Models For Mobile Communications. Proceedings Of Ieee Antennas And Propagation Magazine, 45, 51-82.

Stojmenovic, M. S. I. and Zunic, J. (2002) Dominating Sets And Neighbor Elimination-Based Broadcasting Algorithms In Wireless Networks. Ieee Transaction On Parallel And Distributed Systems, 13, 14 - 25.

The Network Simulator Ns-2, Available From: Http://Www.Isi.Edu/Nsnam/Ns/.

Tseng, Y.-C., Ni, S.-Y., Chen, Y.-S. and Sheu, J.-P. (2002) The Broadcast Storm Problem In A Mobile Ad Hoc Network. Wireless Networks, 8, 153-167.

Tseng, Y.-C., Ni, S.-Y. and Shih, E.-Y. (2003) Adaptive Approaches To Relieving Broadcast Storms In A Wireless Multihop Ad Hoc Networks. Ieee Transaction On Computers, 52, 545557.

Williams, B. and Camp, T. (2002) Comparison Of Broadcasting Techniques For Mobile Ad Hoc Networks. Proceeding Mobihoc. Lausanne, Switzerland, Acm.

$\mathrm{Wu}$, J. and Lou, W. (2003) Forward-Node-SetBased Broadcast In Clustered Mobile Ad Hoc Networks. Wireless Communication And Mobile Computing, 3, 155 - 173.

Zhang, Q. and Agrawal, D. P. (2005) Dynamic Probabilistic Broadcast In Manets. Journal Of Parallel And Distributed Computing, 65, 220-233. 